\title{
Evaluation of a Particle Method for the Ellipsoidal Statistical Bhatnagar-Gross-Krook Equation
}

\author{
Jonathan M. Burt ${ }^{1}$ and Iain D. Boyd ${ }^{2}$ \\ Department of Aerospace Engineering \\ University of Michigan, Ann Arbor, MI 48109
}

\begin{abstract}
A particle method is presented for the numerical simulation of rarefied gas flows, based on the ellipsoidal statistical Bhatnagar-Gross-Krook (ES-BGK) model of the Boltzmann equation. The simulation procedure includes consideration of rotational nonequilibrium, and enforces exact momentum and energy conservation for a mixture involving monatomic and diatomic species. This method is applied to the simulation of a nozzle flow of the type associated with cold-gas spacecraft thrusters, and flowfield characteristics are compared with experimental data as well as results from direct simulation Monte Carlo (DSMC) and Navier-Stokes simulations of the same flow. The ES-BGK method is shown to allow for a relatively high degree of accuracy in transitional flow regimes, while avoiding the intermolecular collision calculations which typically make the DSMC simulation of low Knudsen number flows prohibitively expensive.
\end{abstract}

\section{Introduction}

$\mathrm{I}_{\mathrm{t}}^{\mathrm{n}}$ the design and performance analysis of low-thrust spacecraft propulsion systems, various numerical simulation techniques may be employed to determine efficiency, thrust characteristics, or the potential for plume impingement and contamination on spacecraft surfaces. A particular challenge in the simulation of small thrusters involving a chemically inert neutral gas, such as electro-thermal or cold-gas thrusters, is the accurate consideration of a wide range of Knudsen number regimes. In a typical thruster of this type, gas is expelled through a convergentdivergent (Laval) nozzle into a near vacuum, with subsonic near-equilibrium flow in the convergent section of the nozzle. As the gas accelerates through the divergent section beyond the nozzle throat, a subsonic boundary layer grows along the wall, while a supersonic core-flow region exists around the nozzle centerline. The gas density continues to decrease with downstream distance through the nozzle, and rarefaction effects become more prominent within both the supersonic and subsonic regions. Here the gas velocity distribution begins to diverge significantly from the equilibrium limit, and thermal energy is increasingly distributed non-uniformly among the translational and internal degrees of freedom. Beyond the nozzle exit plane, these nonequilibrium effects continue to increase as the gas rapidly expands and thermal energy is converted into energy associated with bulk motion of the exhaust flow. Rotational freezing occurs due to the large gradients and low collision frequency, and the flow approaches the free molecular limit within a short distance of the nozzle exit, particularly at points far from the nozzle centerline.

The simulation of highly rarefied flows, as described above for the divergent nozzle region and plume, is typically performed using the direct simulation Monte Carlo (DSMC) method of Bird. ${ }^{1}$ This method approximates a numerical solution to the Boltzmann equation - the governing equation for dilute gas flows based on a statistical representation of molecular velocities - by decoupling in time the collision and advection terms in the equation. A large number of particles, each representing a large number of atoms or molecules, are tracked through a computational grid, and are sorted into cells according to their location. During each time step, some fraction of the particles in a cell collide with each other, and probabilistic techniques are used for calculations of individual collisions. All particles are then moved through the grid according to assigned velocities, and particles are created or removed at inflow and outflow boundaries. Finally, macroscopic quantities are sampled by averaging various particle properties in each cell, and the process is then repeated at the next time step.

The DSMC method has been shown to provide accurate solutions for highly rarefied nozzle and plume flows, ${ }^{2}$ and retains validity through the full range of possible Knudsen numbers for a dilute gas. In theory then, DSMC may be applied to the entire flowfield for a small spacecraft thruster as described above. The main obstacle, however, to

\footnotetext{
${ }^{1}$ Graduate student, AIAA student member.

${ }^{2}$ Professor, AIAA associate fellow.
} 
the DSMC simulation of the higher density flow near and upstream of the nozzle throat is the enormous computational cost. There are three major factors which tend to make DSMC prohibitively expensive, even on large parallel machines, for the simulation of relatively high-density flows: First, for accurate simulation the cell size should ideally be much smaller than any characteristic length scale for gradients in macroscopic flow quantities, and nonphysical diffusion effects become increasingly significant as the cell size increases. This generally limits cell dimensions to roughly one mean free path, so that a very large number of cells may be required for the simulation of even small-scale flows when the gas density is high. Second, the temporal decoupling of collision and advection processes is in general a valid approximation only if the time step is no larger than the local mean collision time. This means that for a high density or low Knudsen number flow, a very large number of time steps may be required to reach steady state conditions. These two requirements may be relaxed somewhat when macroscopic gradient length scales are very large compared to the mean free path, so that the flow approaches a near-homogeneous state. However, an additional limiting factor for high density flows cannot be relaxed under near-homogeneous conditions: The collisional process driving a flow toward equilibrium is modeled in DSMC through the probabilistic calculation of individual collisions between pairs of representative particles. The collision frequency increases with the gas density, so that for a fixed set of simulation parameters, a higher density flow corresponds to an increase in the average number of collisions experienced per particle during each time step. Under conditions typical in the convergent nozzle region and around the nozzle throat in a small electro-thermal or cold-gas spacecraft thruster, this last requirement can make DSMC simulation of such flows prohibitively expensive on all but the largest parallel machines.

In practice, these small-scale exhaust flow simulations may be performed using a finite volume representation of the Navier-Stokes (NS) equations in the high density regions where the continuum and quasi-equilibrium assumptions underlying these equations are considered valid. The DSMC method is then used only in the lower density regions where these assumptions break down, and where a DSMC flowfield calculation is feasible using available computational resources. The interface between NS and DSMC regions is modeled either through an uncoupled matching of boundary conditions for two independent simulations, or using a single hybrid code where the interface location may be varied dynamically during the simulation through the evaluation of one or more continuum breakdown parameters. For either approach, the accurate determination of the boundary beyond which the NS solution is invalid may be difficult and expensive. As a result, there is generally some significant tradeoff between overall simulation accuracy and efficiency: Increased accuracy results from a more precise or conservative determination of the proper boundary for the NS domain. This in turn requires a greater computational load, either through an iterative procedure to evaluate the boundary then update the overall flowfield solution, or through an extension of the DSMC domain into regions which may be accurately handled by the NS solver.

This tradeoff between accuracy and efficiency may to some extent be avoided if an alternate simulation approach is used in flowfield regions where the validity of the Navier-Stokes equations is questionable, but the application of DSMC is particularly expensive. This alternate method must be more efficient than DSMC in modeling relatively high density flows, able to account for nonequilibrium phenomena which characterize a transition Knudsen number regime out of range of NS solutions, and in good quantitative agreement with both NS and DSMC for nearequilibrium flows. One such method is presented here, using a particle-based probabilistic solution to the ellipsoidal statistical Bhatnagar-Gross-Krook (BGK) model of the Boltzmann equation.

In recent years, several authors have developed particle methods in which standard DSMC collision operations are replaced by alternate procedures that account for the collisional drive toward equilibrium without modeling individual collisions. ${ }^{3-9}$ In place of collision calculations on pairs of representative particles, some fraction of particles are randomly selected during each time step, and are assigned new velocities according to set distribution functions. In several of these methods, the collision process in each cell within the computational grid is based on the homogeneous form of the BGK equation:

$$
d(n f) / d t=v n\left(f_{\mathrm{e}}-f\right)
$$

Here $f$ is the velocity distribution function for a simple monatomic gas, $f_{\mathrm{e}}$ is a Maxwellian distribution with the same average and variance as $f, n$ is the number density, and $v$ is a characteristic relaxation frequency. This is a simplified linear model of the highly nonlinear homogeneous Boltzmann equation, where the detailed physics of the collision term is ignored in favor of a Maxwell-type molecular model in which the entire distribution function $f$ decays toward equilibrium at a velocity-independent rate. As proposed by Macrossan ${ }^{6}$ and independently implemented by Gallis and Torczynski ${ }^{8}$ and Andries et al., ${ }^{9}$ the method may be modified for better quantitative agreement with the Boltzmann equation as a solution to 


$$
d(n f) / d t=v n\left(f_{\mathrm{G}}-f\right)
$$

where $f_{\mathrm{G}}$ is an anisotropic Gaussian distribution function. This modification to the BGK equation was proposed by Holway ${ }^{10}$ and Cercignani ${ }^{11}$ to correct for the nonphysical constraint in the BGK model of unity Prandtl number, and has recently been shown to satisfy Boltzmann's H-theorem for entropy production. ${ }^{12}$ Following Cercignani, we refer to this as the ellipsoidal statistical BGK (ES-BGK) model.

In the first section of this paper, we extend the methods of Macrossan ${ }^{6}$ and Gallis and Torczynski ${ }^{8}$ for a gas mixture involving monatomic and diatomic species, and describe a procedure to enforce exact momentum and energy conservation while allowing for effects of rotational nonequilibrium. A simple homogeneous test case is then used to verify the accuracy of the proposed rotational relaxation model. Next, the method is applied to a nozzle flow test case involving a small cold-gas thruster with $\mathrm{N}_{2}$ propellant exhausting into a vacuum, and results are compared with DSMC, NS, and experimental data for the same flowfield. We demonstrate relatively good agreement between ES-BGK, DSMC and experimental results, particularly in higher density regions near the nozzle throat, and show that the ES-BGK flowfield solution incorporates some nonequilibrium effects which are also captured by DSMC but neglected in the NS calculation.

\section{Numerical Method}

As discussed above, all procedures unrelated to intermolecular collisions in the ES-BGK particle method are identical to those in DSMC. However, the process of calculating individual representative collisions is replaced by a procedure which involves the resampling of particle properties from predetermined distributions. Consider the ESBGK collision procedure in a cell containing $\mathrm{N}$ particles, where each particle may be assigned to one of several different monatomic or diatomic gas species. We assume vibrational and electronic energy modes are unexcited, and the variable hard sphere (VHS) collision model ${ }^{1}$ is used to approximate the variation of dynamic viscosity $\mu$ with translational temperature $T_{t}$. As a first step in the method, $T_{t}$ is calculated as a function of the average momentum and translational energy of all particles in the cell:

$$
\mathrm{T}_{\mathrm{t}}=\frac{1}{3 \mathrm{k}} \frac{\mathrm{N}}{(\mathrm{N}-1)}\left(\left\langle\mathrm{m} \mathbf{c}^{2}\right\rangle_{a}-\langle\mathrm{m} \mathbf{c}\rangle_{a}^{2} /\langle\mathrm{m}\rangle_{a}\right)
$$

Here $\mathrm{m}$ and $\mathbf{c}$ are the mass and velocity respectively of an individual particle, $\mathrm{k}$ is Boltzmann's constant, the operator \langle\rangle$_{a}$ designates an average over all particles in the cell, and the notation $\mathbf{x}^{2}$ for any vector $\mathbf{x}$ is used to represent $\mathbf{x} \cdot \mathbf{x}$. The coefficient $\mathrm{N} /(\mathrm{N}-1)$ in Eq. (3) corrects for a statistical error which appears when the traditional formula for $\mathrm{T}_{\mathrm{t}}$ based on the velocity distribution is applied to a discrete set of particle velocities. ${ }^{13}$

Using a first order Chapman-Enskog expansion of the ES-BGK equation, it can be shown that the correct relations for heat flux and shear stress are recovered in a near-equilibrium flow if the relaxation frequency is given by

$$
\nu=\operatorname{Pr} \cdot \frac{p}{\mu}
$$

where Pr is the Prandtl number and $p$ is the pressure. From the ideal gas equation of state and the VHS approximation that $\mu$ scales with temperature to some constant power $\omega$, Eq. (4) may be rewritten as

$$
\nu=\operatorname{Pr} \cdot n \mathrm{k}\left(\frac{\mathrm{T}_{\mathrm{ref}}^{\omega}}{\mu_{\mathrm{ref}}}\right) \mathrm{T}_{\mathrm{t}}^{1-\omega}
$$

where $T_{\text {ref }}$ is a reference temperature and $\mu_{\text {ref }}$ is the dynamic viscosity at $T_{\text {ref. }}$ For a simple VHS gas, this viscosity can be calculated as a function of $\omega$ and the molecular collision diameter $\mathrm{d}_{\mathrm{ref}}$ at the reference temperature:

$$
\mu_{\mathrm{ref}}=\frac{30\left(\mathrm{mkT}_{\mathrm{ref}} / \pi\right)^{1 / 2}}{4(5-2 \omega)(7-2 \omega) \mathrm{d}_{\mathrm{ref}}^{2}}
$$

American Institute of Aeronautics and Astronautics Approved for public release; distribution unlimited. 
Note that in most cases the value of $d_{\text {ref }}$ is itself determined from experimental measurements of $\mu_{\text {ref. }}$ Eq. (6) is useful however in a hybrid code (discussed below) involving both DSMC and the method proposed here, as reference collision diameters are among the required input parameters for DSMC collision calculations. A simple approximate extension of Eq. (6) to a gas mixture may be obtained by summing the product of $\mu_{\text {ref }}$ and the timeaveraged species mass fraction in the cell over all gas species.

Once the relaxation frequency $v$ is known, Eq. (2) may be integrated to give the distribution function $f$ after some finite time step $\Delta \mathrm{t}$ as a linear combination of the initial distribution $f(0)$ and a corresponding anisotropic Gaussian distribution $f_{\mathrm{G}}$.

$$
f(\Delta \mathrm{t})=f(0) \exp (-\nu \Delta \mathrm{t})+f_{\mathrm{G}}(1-\exp (-\nu \Delta \mathrm{t}))
$$

From Eq. (7), we determine the number of particles $\mathrm{N}_{\mathrm{v}}$ selected for reassignment of velocity components as

$$
\mathrm{N}_{\mathrm{v}}=\text { floor }[\mathrm{N}(1-\exp (-\nu \Delta \mathrm{t}))+R]
$$

where $v$ is evaluated using Eq. (5), $R$ is a random number in $[0,1]$ and the operator "floor" rounds to the nearest smaller integer. Note that a time-averaged value of the number density $n$ should be used in Eq. (5) to avoid an error associated with statistical fluxuations in the calculated relaxation frequency. Once $\mathrm{N}_{\mathrm{v}}$ is known, this number of unique particles is then randomly selected and assigned to a list for velocity resampling. (In order to reduce calls to the random number generator, if $\mathrm{N}_{\mathrm{v}}>1 / 2 \mathrm{~N}$ then $\mathrm{N}-\mathrm{N}_{\mathrm{v}}$ randomly chosen particles are removed from a list which initially includes all particles in the cell, and the remaining particles are used for resampling.) In the special case where $\mathrm{N}_{\mathrm{v}}=1$, momentum conservation requires that the velocity of the selected particle remains constant, so that $\mathrm{N}_{\mathrm{v}}$ values of zero and one are equivalent during the resampling procedure. To correct for the resulting error, if $\mathrm{N}_{\mathrm{v}}$ is calculated as one through Eq. (8) then an additional random number is generated and $\mathrm{N}_{\mathrm{v}}$ is set with equal probability to either zero or two.

After $\mathrm{N}_{\mathrm{v}}$ particles have been selected, all velocity components of these particles are resampled from a normalized Maxwellian distribution. Each velocity component is set to either

$$
\mathrm{c}_{\mathrm{i}}^{*}=\cos \left(2 \pi R_{1}\right) \sqrt{-\ln \left(R_{2}\right) / \mathrm{m}} \quad \text { or } \quad \mathrm{c}_{\mathrm{i}}^{*}=\sin \left(2 \pi R_{1}\right) \sqrt{-\ln \left(R_{2}\right) / \mathrm{m}}
$$

where the subscript $\mathrm{i}$ denotes the direction, $\mathrm{m}$ is the particle mass, and $R_{1}$ and $R_{2}$ are random numbers between 0 and 1. Two statistically independent values of $\mathrm{c}_{\mathrm{i}}{ }^{*}$ may be obtained if Eqs. (9) are evaluated for the same values of $R_{1}$ and $R_{2}$, so that only a single random number must be generated per velocity component. Following Gallis and Torczynski, ${ }^{8}$ we then modify all resampled velocities to new values $\mathrm{c}_{\mathrm{i}}{ }^{(1)}$ which conform to an anisotropic Gaussian distribution:

where

$$
\mathrm{c}_{\mathrm{i}}^{(1)}=\mathrm{S}_{\mathrm{ij}} \mathrm{c}_{\mathrm{j}}^{*}
$$

$$
\mathrm{S}_{\mathrm{ij}}=\delta_{\mathrm{ij}}-\frac{1-\operatorname{Pr}}{2 \operatorname{Pr}}\left[\frac{1}{\mathrm{kT}_{\mathrm{t}}} \frac{\mathrm{N}}{\mathrm{N}-1}\left(\left\langle\mathrm{mc}_{\mathrm{i}} \mathrm{c}_{\mathrm{j}}\right\rangle_{a}-\left\langle\mathrm{mc}_{\mathrm{i}}\right\rangle_{a}\left\langle\mathrm{mc}_{\mathrm{j}}\right\rangle_{a} /\langle\mathrm{m}\rangle_{a}\right)-\delta_{\mathrm{ij}}\right]
$$

Here $\delta_{\mathrm{ij}}$ is the Kronecker delta, $\mathrm{c}_{\mathrm{i}}$ and $\mathrm{c}_{\mathrm{j}}$ are particle velocity components before resampling, $\mathrm{T}_{\mathrm{t}}$ is evaluated using Eq. (3), and the superscript (1) indicates a newly reassigned value.

To account for the effect of collisions on the rotational energy distribution among diatomic gas species, we define a rotational relaxation frequency $v_{\mathrm{r}}$ such that, in a homogeneous gas, the instantaneous cell-averaged rotational temperature $\mathrm{T}_{\mathrm{r}}$ will vary as

$$
d \mathrm{~T}_{\mathrm{r}} / d t=-v_{\mathrm{r}}\left(\mathrm{T}_{\mathrm{r}}-\mathrm{T}_{\mathrm{eq}}\right)
$$

Here $T_{\text {eq }}$ is the equilibrium temperature to which both $T_{r}$ and $T_{t}$ converge after a long period of time, and $T_{r}$ is given by 


$$
\mathrm{T}_{\mathrm{r}}=\frac{2}{\mathrm{k}}\left\langle E_{\mathrm{r}}\right\rangle_{\mathrm{a}} /\left\langle\zeta_{\mathrm{r}}\right\rangle_{\mathrm{a}}
$$

The above symbols $E_{\mathrm{r}}$ and $\zeta_{\mathrm{r}}$ denote rotational energy and rotational degrees of freedom, respectively, for an individual particle. The equilibrium temperature can be expressed as a weighted sum of the rotational and translational temperatures, such that

$$
\zeta_{\mathrm{t}, \mathrm{eff}} \mathrm{T}_{\mathrm{t}}+\left\langle\zeta_{\mathrm{r}}\right\rangle_{\mathrm{a}} \mathrm{T}_{\mathrm{r}}=\left(\zeta_{\mathrm{t}, \mathrm{eff}}+\left\langle\zeta_{\mathrm{r}}\right\rangle_{\mathrm{a}}\right) \mathrm{T}_{\mathrm{eq}}
$$

where $\zeta_{\mathrm{t}, \text { eff }}$ is the effective number of translational degrees of freedom for all particles in the cell. If all $\mathrm{N}$ particles are together considered as an isolated system, then it follows from Eqs. (3) and (13) and energy conservation arguments that

$$
\frac{d}{d t}\left[\langle\mathrm{mc}\rangle_{\mathrm{a}}^{2} /\langle\mathrm{m}\rangle_{\mathrm{a}}+3\left(\frac{\mathrm{N}-1}{\mathrm{~N}}\right) \mathrm{k} \mathrm{T}_{\mathrm{t}}+\left\langle\zeta_{\mathrm{r}}\right\rangle_{\mathrm{a}} \mathrm{k} \mathrm{T}_{\mathrm{r}}\right]=0
$$

while momentum and mass conservation require that the first term within the brackets be constant in time as well. By comparing Eqs. (14) and (15) and noting that $\mathrm{T}_{\mathrm{eq}}$ is a time invariant quantity, we can show that there are effectively $3(\mathrm{~N}-1) / \mathrm{N}$ translational degrees of freedom in the cell, so that the equilibrium temperature may be given from Eq. (14) as

$$
\mathrm{T}_{\mathrm{eq}}=\frac{3((\mathrm{~N}-1) / \mathrm{N}) \mathrm{T}_{\mathrm{t}}+\left\langle\zeta_{\mathrm{r}}\right\rangle_{\mathrm{a}} \mathrm{T}_{\mathrm{r}}}{3(\mathrm{~N}-1) / \mathrm{N}+\left\langle\zeta_{\mathrm{r}}\right\rangle_{\mathrm{a}}}
$$

Note that the arguments used to derive Eq. (16) make no assumptions regarding how the translational and rotational temperatures relax toward the equilibrium state, so that this equation should also be valid for DSMC and other methods for which a discrete set of particle velocities is used to describe the velocity distribution of a gas in rotational nonequilibrium. As there are exactly three translational degrees of freedom for any real gas under consideration here, this derivation reveals the existence of a deterministic error which scales as $(\mathrm{N}-1) / \mathrm{N}$ and may significantly influence calculated bulk flow properties when the average number of particles per cell is very small. To the authors' knowledge no previous references to this error source exist in the literature, although the upper bound of the resulting error should be relatively small (less than 5\%) when particle numerical weights are set such that over 20 particles are assigned to each cell, as is standard practice in DSMC.

The rotational relaxation frequency $v_{\mathrm{r}}$ shown in Eq. (12) is defined here as the frequency of inelastic collisions, given as the ratio of the collision frequency $F_{\text {coll }}$ to the rotational collision number $Z_{\mathrm{r}}$. To determine $F_{\text {coll }}$, consider a cell containing particles of $\mathrm{N}_{\text {spec }}$ different species, each with a mass $\mathrm{m}_{\mathrm{i}}$, VHS reference collision diameter $\mathrm{d}_{\mathrm{ref}, \mathrm{i}}$ and number density $n_{\mathrm{i}}$ averaged over a large number of time steps. Assuming a near-equilibrium flow, we can efficiently calculate the collision frequency as

$$
F_{\text {coll }}=\frac{4}{n} \sqrt{\frac{\mathrm{kT}_{\mathrm{ref}}}{2 \pi}}\left(\frac{\mathrm{T}_{\mathrm{t}}}{\mathrm{T}_{\mathrm{ref}}}\right)^{1-\omega} \sum_{\mathrm{i}=1}^{\mathrm{N}_{\text {spec }}} \sum_{\mathrm{j}=1}^{\mathrm{i}} n_{\mathrm{i}} n_{\mathrm{j}} \mathrm{A}_{\mathrm{ij}}\left(2-\delta_{\mathrm{ij}}\right)
$$

where

$$
\mathrm{A}_{\mathrm{ij}}=\frac{\pi}{4}\left(\mathrm{~d}_{\mathrm{ref}, \mathrm{i}}+\mathrm{d}_{\mathrm{ref}, \mathrm{j}}\right)^{2} \sqrt{\frac{\mathrm{m}_{\mathrm{i}}+\mathrm{m}_{\mathrm{j}}}{\mathrm{m}_{\mathrm{i}} \mathrm{m}_{\mathrm{j}}}} \text { and } n=\sum_{\mathrm{i}=1}^{\mathrm{N}_{\mathrm{sppec}}} n_{\mathrm{i}} .
$$

Note that no implied summations are used in the index notation of Eq. (17).

The rotational collision number $Z_{\mathrm{r}}$ is calculated using a modified form of a formula given by Parker. ${ }^{14}$

$$
\mathrm{Z}_{\mathrm{r}}=\frac{3 / 5 \mathrm{Z}_{\mathrm{r}}^{\infty}}{1+\left(\pi^{1 / 2} / 2\right)\left(\mathrm{T}^{*} / \mathrm{T}_{\mathrm{eq}}\right)^{1 / 2}+\left(\pi+\pi^{2} / 4\right)\left(\mathrm{T}^{*} / \mathrm{T}_{\mathrm{eq}}\right)}
$$


The above constants $Z_{\mathrm{r}}^{\infty}$ and $\mathrm{T}^{*}$ are determined for $\mathrm{N}_{2}$ as $Z_{\mathrm{r}}^{\infty}=23.0$ and $\mathrm{T}^{*}=91.5 \mathrm{~K}^{1}$ As a modification to Parker's formula, the equilibrium temperature $T_{\text {eq }}$ is used here in place of $T_{t}$ following observations of Boyd ${ }^{15}$ that, for a single intermolecular collision, $Z_{\mathrm{r}}$ tends to vary as a function of both the translational and rotational collision energies. The $3 / 5$ factor in the numerator, also not in Parker's original formula, accounts for the fact that $Z_{\mathrm{r}}$ is defined here to characterize the rate at which $T_{r}$ relaxes toward $T_{\text {eq }}$, not toward $T_{t}$ as used by Parker and others. ${ }^{1}$

By analogy with Eq. (8), Eq. (12) can be integrated in time to give an initial estimate of the number of particles $\mathrm{N}_{\mathrm{r}}$ for which to resample rotational energy as

$$
\mathrm{N}_{\mathrm{r}} \approx \mathrm{N}\left(1-\exp \left(-\frac{F_{\text {coll }}}{\mathrm{Z}_{\mathrm{r}}} \Delta \mathrm{t}\right)\right)
$$

where $F_{\text {coll }}$ and $Z_{\mathrm{r}}$ are evaluated using Eqs. (17) and (18) respectively. (The exact determination of $\mathrm{N}_{\mathrm{r}}$ requires a more involved procedure, and is discussed in detail below.) In our implementation, $\mathrm{N}_{\mathrm{r}}$ particles are randomly selected from the list of $\mathrm{N}_{\mathrm{v}}$ particles which have already been tagged for velocity resampling, and the rotational energy $E_{\mathrm{r}}$ of each selected diatomic particle is reassigned to a normalized value

$$
E_{\mathrm{r}}^{(1)}=-\ln (R)
$$

where a unique random number $R \in[0,1]$ is used for each particle.

To avoid random walk errors and permit overall accuracy for a near-equilibrium flow comparable to that of either DSMC or NS simulations, we desire for both momentum and energy to be exactly conserved during the resampling procedures. It follows that all reassigned velocities $\mathbf{c}^{(1)}$ and rotational energies $E_{\mathrm{r}}^{(1)}$ must be modified to final values $\mathbf{c}^{(2)}$ and $E_{\mathrm{r}}^{(2)}$ such that

$$
\left\langle\mathrm{mc}^{(2)}\right\rangle_{\mathrm{v}}=\left\langle\mathrm{m} \mathbf{c}^{(0)}\right\rangle_{\mathrm{v}}
$$

and

$$
\frac{1}{2} \mathrm{~N}_{\mathrm{v}}\left\langle\mathrm{m}\left(\mathbf{c}^{(2)}\right)^{2}\right\rangle_{\mathrm{v}}+\mathrm{N}_{\mathrm{r}}\left\langle E_{\mathrm{r}}^{(2)}\right\rangle_{\mathrm{r}}=\frac{1}{2} \mathrm{~N}_{\mathrm{v}}\left\langle\mathrm{m}\left(\mathbf{c}^{(0)}\right)^{2}\right\rangle_{\mathrm{v}}+\mathrm{N}_{\mathrm{r}}\left\langle E_{\mathrm{r}}^{(0)}\right\rangle_{\mathrm{r}}
$$

where the operators \langle\rangle$_{\mathrm{V}}$ and \langle\rangle$_{\mathrm{r}}$ denote averages over all particles used in the resampling of velocity and rotational energy respectively. Further, to satisfy energy equipartition at the equilibrium limit, we require that translational and rotational temperatures based on reassigned values be equal. This condition can be written as

$$
\frac{1}{3 \mathrm{k}} \frac{\mathrm{N}_{\mathrm{v}}}{\left(\mathrm{N}_{\mathrm{v}}-1\right)}\left(\left\langle\mathrm{m}\left(\mathbf{c}^{(2)}\right)^{2}\right\rangle_{\mathrm{v}}-\left\langle\mathrm{m} \mathbf{c}^{(2)}\right\rangle_{\mathrm{v}}^{2} /\langle\mathrm{m}\rangle_{\mathrm{v}}\right)=\frac{2}{\mathrm{k}}\left\langle E_{\mathrm{r}}^{(2)}\right\rangle_{\mathrm{r}} /\left\langle\zeta_{\mathrm{r}}\right\rangle_{\mathrm{r}}
$$

where $\zeta_{\mathrm{r}}$ is the number of rotational degrees of freedom for an individual particle. For each of the $\mathrm{N}_{\mathrm{v}}$ particles selected for velocity resampling, the final velocity is calculated as

$$
\mathbf{c}^{(2)}=\boldsymbol{B}+C\left(\mathbf{c}^{(1)}-\left\langle\mathrm{m} \mathbf{c}^{(1)}\right\rangle_{\mathrm{v}} /\langle\mathrm{m}\rangle_{\mathrm{v}}\right)
$$

while each of the $\mathrm{N}_{\mathrm{r}}$ particles with resampled rotational energies is given a final value of

$$
E_{\mathrm{r}}^{(2)}=D E_{\mathrm{r}}^{(1)} .
$$

By algebraic manipulation of Eqs. (21) through (25), we can show that momentum conservation, energy conservation and energy equipartition are all satisfied if the vector $\boldsymbol{B}$ and scalar constants $C$ and $D$ are chosen as follows:

$$
\boldsymbol{B}=\left\langle\mathrm{m} \mathbf{c}^{(0)}\right\rangle_{\mathrm{v}} /\langle\mathrm{m}\rangle_{\mathrm{v}}
$$




$$
\begin{gathered}
C=\left[\left(\frac{3\left(\mathrm{~N}_{\mathrm{v}}-1\right)}{3\left(\mathrm{~N}_{\mathrm{v}}-1\right)+\mathrm{N}_{\mathrm{r}}\left\langle\zeta_{\mathrm{r}}\right\rangle_{\mathrm{r}}}\right)\left(\frac{\left\langle\mathrm{m}\left(\mathbf{c}^{(0)}\right)^{2}\right\rangle_{\mathrm{v}}-\left\langle\mathrm{m} \mathbf{c}^{(0)}\right\rangle_{\mathrm{v}}^{2} /\langle\mathrm{m}\rangle_{\mathrm{v}}+2\left(\mathrm{~N}_{\mathrm{r}} / \mathrm{N}_{\mathrm{v}}\right)\left\langle E_{\mathrm{r}}^{(0)}\right\rangle_{\mathrm{r}}}{\left\langle\mathrm{m}\left(\mathbf{c}^{(1)}\right)^{2}\right\rangle_{\mathrm{v}}-\left\langle\mathrm{m} \mathbf{c}^{(1)}\right\rangle_{\mathrm{v}}^{2} /\langle\mathrm{m}\rangle_{\mathrm{v}}}\right)\right]^{1 / 2} \\
D=\left(\frac{\mathrm{N}_{\mathrm{v}}\langle\zeta\rangle_{\mathrm{r}}}{3\left(\mathrm{~N}_{\mathrm{v}}-1\right)+\mathrm{N}_{\mathrm{r}}\left\langle\zeta_{\mathrm{r}}\right\rangle_{\mathrm{r}}}\right)\left(\frac{\left\langle\mathrm{m}\left(\mathbf{c}^{(0)}\right)^{2}\right\rangle_{\mathrm{v}}-\left\langle\mathrm{m} \mathbf{c}^{(0)}\right\rangle_{\mathrm{v}}^{2} /\langle\mathrm{m}\rangle_{\mathrm{v}}+2\left(\mathrm{~N}_{\mathrm{r}} / \mathrm{N}_{\mathrm{v}}\right)\left\langle E_{\mathrm{r}}^{(0)}\right\rangle_{\mathrm{r}}}{2\left\langle E_{\mathrm{r}}^{(1)}\right\rangle_{\mathrm{r}}}\right)
\end{gathered}
$$

From the definition of rotational temperature indicated by Eq. (13), the right hand side of Eq. (28) may be expressed as a ratio of temperatures: The denominator gives the rotational temperature based on values of $E_{\mathrm{r}}^{(1)}$, while the numerator gives the temperature corresponding to the conditions of Eq. (23). Thus, Eq. (28) may be used to show that the temperature $T^{(2)}$ based on resampled and renormalized values of either particle velocity $\mathbf{c}^{(2)}$ or rotational energy $E_{\mathrm{r}}^{(2)}$ can be given as

$$
\mathrm{T}^{(2)}=\left(\frac{\mathrm{N}_{\mathrm{v}} / \mathrm{k}}{3\left(\mathrm{~N}_{\mathrm{v}}-1\right)+\mathrm{N}_{\mathrm{r}}\left\langle\zeta_{\mathrm{r}}\right\rangle_{\mathrm{r}}}\right)\left(\left\langle\mathrm{m}\left(\mathbf{c}^{(0)}\right)^{2}\right\rangle_{\mathrm{v}}-\left\langle\mathrm{m} \mathbf{c}^{(0)}\right\rangle_{\mathrm{v}}^{2} /\langle\mathrm{m}\rangle_{\mathrm{v}}+2\left(\frac{\mathrm{N}_{\mathrm{r}}}{\mathrm{N}_{\mathrm{v}}}\right)\left\langle E_{\mathrm{r}}^{(0)}\right\rangle_{\mathrm{r}}\right)
$$

As particles are randomly chosen for velocity or rotational energy resampling, the averaged quantities in Eq. (29) will, on average, equal the corresponding quantities averaged over all $\mathrm{N}$ particles in the cell. If we denote the equivalent temperature based on cell-averaged values as $T_{a}{ }^{(2)}$, then it follows from substitution of Eqs. (3) and (13) into Eq. (29) that

$$
\mathrm{T}_{\mathrm{a}}^{(2)}=\frac{3\left(\left(\mathrm{~N}_{\mathrm{v}}-1\right) / \mathrm{N}_{\mathrm{r}}\right) \mathrm{T}_{\mathrm{t}}+\left\langle\zeta_{\mathrm{r}}\right\rangle_{\mathrm{a}} \mathrm{T}_{\mathrm{r}}}{3\left(\mathrm{~N}_{\mathrm{v}}-1\right) / \mathrm{N}_{\mathrm{r}}+\left\langle\zeta_{\mathrm{r}}\right\rangle_{\mathrm{a}}}
$$

As the temperature $\mathrm{T}_{\mathrm{a}}{ }^{(2)}$ is generally not equal to the equilibrium temperature $\mathrm{T}_{\mathrm{eq}}$ determined from Eq. (16), the procedure used to calculate $\mathrm{N}_{\mathrm{r}}$ must be based on a relaxation frequency other than the true characteristic frequency of rotational relaxation $v_{\mathrm{r}}=F_{\text {coll }} / \mathrm{Z}_{\mathrm{r}}$. A correction factor $\beta$ is therefore introduced as the ratio of this required relaxation frequency to $v_{\mathrm{r}}$, so that in a homogeneous adiabatic flow,

$$
d \mathrm{~T}_{\mathrm{r}} / d t=-\beta v_{\mathrm{r}}\left(\mathrm{T}_{\mathrm{r}}-\mathrm{T}_{\mathrm{a}}^{(2)}\right)
$$

From a comparison of Eqs. (12) and (31), it follows that

$$
\beta=\left(\mathrm{T}_{\mathrm{r}}-\mathrm{T}_{\mathrm{eq}}\right) /\left(\mathrm{T}_{\mathrm{r}}-\mathrm{T}_{\mathrm{a}}^{(2)}\right)
$$

Substituting Eqs. (16) and (30) into Eq. (32), we find

$$
\beta=\frac{3+\left\langle\zeta_{\mathrm{r}}\right\rangle_{\mathrm{a}} \mathrm{N}_{\mathrm{r}}^{*} /\left(\mathrm{N}_{\mathrm{v}}-1\right)}{3+\left\langle\zeta_{\mathrm{r}}\right\rangle_{\mathrm{a}} \mathrm{N} /(\mathrm{N}-1)}
$$

where $\mathrm{N}_{\mathrm{r}}{ }^{*}$ is an approximation to the integer value $\mathrm{N}_{\mathrm{r}}$ based on an exact solution to Eq. (31). If Eq. (31) is integrated over the time step interval $\Delta t$, then the same reasoning used to find $\mathrm{N}_{\mathrm{v}}$ by Eq. (8) may be used to calculate $\mathrm{N}_{\mathrm{r}}{ }^{*}$ as

$$
\mathrm{N}_{\mathrm{r}}^{*}=\mathrm{N}\left(1-\exp \left(-\beta \frac{F_{\text {coll }}}{\mathrm{Z}_{\mathrm{r}}} \Delta \mathrm{t}\right)\right)
$$

Note from Eqs. (33) and (34) that $\beta$ and $\mathrm{N}_{\mathrm{r}}{ }^{*}$ cannot be independently determined, but must instead be calculated simultaneously using an iterative method. In the implementation proposed here, $\beta$ is initially set to one and $\mathrm{N}_{\mathrm{r}}{ }^{*}$ is found by Eq. (34), after which $\beta$ and $\mathrm{N}_{\mathrm{r}}{ }^{*}$ are updated using Eqs. (33) and (34) respectively. This procedure is repeated four additional times, after which the number of particles to select for rotational energy resampling is 
computed as $\mathrm{N}_{\mathrm{r}}=$ floor $\left[\mathrm{N}_{\mathrm{r}}{ }^{*}+R\right]$ where $R$ is a random number in $[0,1]$. This simple "brute force" approach is found to give a reasonable balance of accuracy and efficiency over a wide range of conditions, and more complicated iterative schemes with faster convergence should not generally be required due to the insignificant contribution of this procedure to the total computational expense.

\section{Homogeneous Rotational Relaxation}

To verify the accuracy of the procedure described above for rotational relaxation, this procedure is implemented in a modified version of the DSMC code $\mathrm{MONACO}^{16}$ and applied to a zero-dimensional test case based on simulations of Bird. ${ }^{1}$ An isolated control volume of $\mathrm{N}_{2}$ gas is given initial conditions involving translational equilibrium at $500 \mathrm{~K}$ and a rotational temperature of $0 \mathrm{~K}$. The simulation domain consists of a single cell with specularly reflecting walls and 10,000 particles, and the time step is set to $1 / 2$ of the initial mean collision time. The rotational collision number is found from Eqs. (16) and (18) to hold a constant value of $Z_{\mathrm{r}}=4.31$, so that the rotational and translational temperatures will vary in time according to analytical expressions similar to those derived by Bird. ${ }^{1}$

$$
\mathrm{T}_{\mathrm{r}}=300\left(1-\exp \left(-\frac{\mathrm{t}}{4.31 \tau_{\text {coll }}}\right)\right), \mathrm{T}_{\mathrm{t}}=300+200 \exp \left(-\frac{\mathrm{t}}{4.31 \tau_{\text {coll }}}\right)
$$

Here both temperatures are given in $\mathrm{K}, \mathrm{t}$ is the total elapsed time and $\tau_{\text {coll }}$ is a characteristic mean collision time. The variable $\tau_{\text {coll }}$ may be defined in terms of the instantaneous mean collision frequency $F_{\text {coll }}$ as

$$
\tau_{\text {coll }}=\mathrm{t}\left[\int_{0}^{\mathrm{t}} F_{\text {coll }}\left(\mathrm{t}^{\prime}\right) d \mathrm{t}^{\prime}\right]^{-1}
$$

where $F_{\text {coll }}$ is calculated for a VHS gas using Eq. (17).

Figure (1) shows the variation in both $T_{r}$ and $T_{t}$ as functions of the normalized time $t / \tau_{\text {coll }}$. Results from the ESBGK simulation are presented along with results from a DSMC simulation under identical conditions, and theoretical curves based on Eqs. (35) are also shown for comparison. For consistency, a constant rotational collision number of 4.31 is used in the DSMC simulation. The ES-BGK data points are found to be in close agreement with both theory and DSMC, while a slight underprediction is observed in the relaxation rate from the ES-BGK simulation. This discrepancy can be traced to the fact that only a fraction of particles are chosen for velocity resampling during each time step. If the artificial condition $\mathrm{N}_{\mathrm{v}}=\mathrm{N}$ is imposed, then nearly perfect agreement is found between the ES-BGK and theoretical curves of Fig. (1). As discussed by Bird, ${ }^{1}$ even DSMC results for homogeneous rotational relaxation with constant $Z_{\mathrm{r}}$ can only be approximated by an analytical solution, and the scale of the disagreement between DSMC and theoretical curves is comparable to that between theory and ES-BGK. Both DSMC and ES-BGK results have some dependence on the VHS temperature exponent $\omega$, so by comparison with DSMC we can conclude that rotational relaxation is modeled with acceptable accuracy by the method proposed here.

\section{Nozzle Flow Simulation}

Few experimental studies are available in the literature involving the measurement of flowfield characteristics within a nozzle of the type employed in cold-gas spacecraft thrusters. To the authors' knowledge, the most detailed is that of Rothe, ${ }^{17}$ where an electron beam technique was used to measure density and rotational temperature for the flow of $\mathrm{N}_{2}$ through a very small nozzle into a vacuum chamber. Both NS and DSMC simulations of the internal nozzle flow have been performed by Chung et al. ${ }^{18}$ In the DSMC simulation by these authors, the computational domain is restricted to the lower density regions beyond the nozzle throat, with inflow conditions at the throat based on a NS simulation of the flow within the convergent section of the nozzle. Generally good agreement is found between DSMC results and experimental data, while a NS simulation of the entire nozzle flow gives relatively poor agreement with the experiment in areas downstream of the throat where nonequilibrium effects become significant.

To evaluate the accuracy of the ES-BGK approach presented here, we perform ES-BGK and DSMC simulations for the divergent nozzle and nearfield plume flow investigated by Rothe. ${ }^{17}$ Identical grids, boundary conditions and numerical weights are used for the two simulations, with the same grid geometry and inflow conditions at the nozzle throat employed in the DSMC simulation of Chung et al. ${ }^{18}$ As the ES-BGK particle method is based on assumptions

American Institute of Aeronautics and Astronautics Approved for public release; distribution unlimited. 
of near-equilibrium flow, we can investigate the applicability of the method to higher Knudsen number regimes by comparing ES-BGK results with both DSMC and experimental data.

Following Chung et al. we use a VHS temperature exponent $(\omega)$ of 0.24 and assume fully diffuse reflection for particle collisions with the nozzle wall. These authors investigated a number of different models for translational energy transfer during particle-wall collisions, and found best agreement with experimental data when all collisions are assumed diffuse, with a $10 \%$ probability of thermal accommodation to the wall temperature of $300 \mathrm{~K}$. Energy exchange between translational and rotational modes was calculated using a detailed model which allows the exchange probability to vary as a function of the total incident energy of the colliding particle. As noted by these authors, a lack of experimental measurements on the interaction of $\mathrm{N}_{2}$ gas with solid graphite (the nozzle wall material) leaves some doubt as to the validity of this energy exchange model. A more commonly accepted procedure for modeling rotational energy transfer during particle-wall collisions in DSMC involves an assumption that particle rotational energy is resampled from an equilibrium distribution at the wall temperature with some uniform probability. Therefore, as a simplification to the procedure described by Chung et al., we assume that $10 \%$ of particle-wall collisions involve full accommodation of both translational and rotational energy to the wall temperature, and the remaining $90 \%$ of these collisions involve adiabatic diffuse reflection. In the latter type of collision, the post-collision direction of the particle trajectory is randomly assigned according to the equilibrium velocity distribution for particles which pass through a plane, while the incident values of both translational and rotational energy are retained.

Axisymmetric ES-BGK and DSMC simulations are performed on a $1.4 \mathrm{GHz}$ AMD Athlon cluster, with static domain decomposition among eight separate tasks. Each calculation is run for 24 hours, with roughly 15 million particles at steady state and an unstructured grid of about 41,000 triangular cells scaled according to the local mean free path. The grid geometry is shown in Fig. (2) following that given by Chung et al. Not visible in the figure is the wall curvature at the throat, where the radius of curvature is one half the throat radius of $2.55 \mathrm{~mm}$. The nozzle divergence angle is $20^{\circ}$, the exit-to-throat area ratio is 66 , and the simulation domain extends $14 \mathrm{~mm}$ downstream of the exit plane. To avoid unphysical flow characteristics beyond the exit plane due to the small domain size, we neglect the effect of ambient pressure in the vacuum chamber and use outflow boundaries within the plume region. Results for both ES-BGK and DSMC simulations are shown in Figs. (3) through (10).

\section{Simulation Results}

Mach number contours for both simulations are shown in Fig. (3), where the top half and bottom half of the figure give ES-BGK and DSMC results, respectively. Excellent agreement between the two methods is found in the area around the nozzle throat. In both simulations, a significant reduction in Mach number is found toward the centerline in this region, as results from a weak compression wave due to the curvature of streamlines at the throat. ${ }^{18}$ Mach number contours along the nozzle wall are also nearly identical, and both results show a termination of the sonic line at the nozzle lip due to the rapid acceleration expected at the lip in any highly underexpanded nozzle flow. However, prominent differences between the two results appear along the centerline beyond a point about $1 \mathrm{~cm}$ downstream of the throat. As the degree of rarefaction increases with downstream distance, the centerline Mach number gradient in the DSMC simulation becomes significantly greater than in the ES-BGK simulation, so that the intersection of the Mach 4 contour line with the axis occurs roughly $2.6 \mathrm{~cm}$ further upstream in DSMC than in ESBGK results. This difference in Mach number is likely a result of the unphysical lack of velocity dependence in the collisional relaxation rate of Eq. (2), which should correspond to some reduction in the streamwise temperature gradient within areas of low collision frequency. The difference between the results is however largely restricted to a narrow region along the axis, and very good agreement between the two sets of contours is still found well downstream of the throat at points further from the centerline. Note that no contour lines are shown within the plume backflow region beyond the nozzle lip, due to the small sample size in this very low density region.

Contours of density are shown in Fig. (4), again with ES-BGK results in the top half and DSMC results in the bottom half of the figure. All values are normalized by the stagnation density $\rho_{\mathrm{o}}=5.323 \times 10^{-3} \mathrm{~kg} / \mathrm{m}^{3}$. As in Fig. (3) we find excellent agreement around the nozzle throat and further downstream in regions far from the centerline. Both results show an off-axis local maximum in the density profile just downstream of the throat, which is consistent with a weak compression wave described by Chung et al. ${ }^{18}$ and mentioned above in the discussion of Mach number contours. As above, the greatest difference between the two results is found along the axis at points far from the throat. Here there is a noticeable increase in the ES-BGK expansion rate relative to that of DSMC, so that at a given point on the axis the density is lower in the ES-BGK results.

This trend is also found in Fig. (5), which shows the normalized density variation along the centerline. The axial coordinate $\mathrm{x}$ in the plot is normalized by the throat radius $\mathrm{R}_{\mathrm{t}}=2.55 \mathrm{~mm}$, and a dotted vertical line gives the location of the nozzle exit plane. Here results for the ES-BGK and DSMC simulations are plotted against those for DSMC

\section{9}

American Institute of Aeronautics and Astronautics Approved for public release; distribution unlimited. 
and NS simulations of Chung et al. ${ }^{18}$ and the experimental data points of Rothe. ${ }^{17}$ For $\mathrm{x} / \mathrm{R}_{\mathrm{t}}<10$ very similar centerline density profiles are found for the two simulations presented here, and a slight difference is observed between these and the DSMC and NS contours given by Chung et al. The reason for this discrepancy is not clear, but two likely possibilities are some difference in grid resolution within this region between the two sets of simulations, or a discretization of flow conditions at the nozzle throat from the use of a finite number of uniform inflow boundaries. In general, the ES-BGK result agrees very well with both DSMC density profiles and the experimental values. We find a significantly higher overall accuracy here for ES-BGK than for NS, particularly far downstream of the throat where density is slightly underpredicted by ES-BGK but greatly overpredicted in the NS simulation.

Figure (6) shows the variation in translational and rotational temperature along the nozzle centerline for ES-BGK and both DSMC simulations, along with the NS centerline temperature and rotational temperature values of Rothe. All temperatures here are normalized by the stagnation temperature $T_{o}=300 \mathrm{~K}$. In comparing the two temperature profiles from the ES-BGK simulation, note that the rotational temperature is uniformly higher than the translational temperature, and that the difference between the two values increases with downstream distance. This trend is also observed in both sets of DSMC results, and demonstrates the rotational energy lag expected in rarefied expansion flows due to a finite rotational relaxation time. The quantitative level of agreement between the experimental measurements and ES-BGK results is in general comparable to that between the experiment and DSMC, although the ES-BGK rotational temperature near the nozzle exit is significantly higher than that found using the other methods.

Density profiles across a radial plane near the nozzle exit are shown in Fig. (7). This plane is defined by the axial location $\mathrm{x} / \mathrm{R}_{\mathrm{t}}=18.7$, and radial coordinates $\mathrm{y}$ are normalized by the local nozzle radius $\mathrm{R}_{\mathrm{w}}$. As discussed above, the correlation between ES-BGK and DSMC results in this region decreases significantly toward the nozzle centerline, where the ES-BGK density is about 7\% lower than that found for either DSMC simulation. Further from the centerline, the agreement between ES-BGK and DSMC results increases considerably, with nearly identical values found at points close to the nozzle wall. At all points along this plane, ES-BGK density values are uniformly lower than those found experimentally, while the density values extracted from the NS solution are significantly greater than the experimental data. Except for a small region roughly half way between the axis and nozzle wall, we find the ES-BGK density values to more closely approximate measured values than do results from the NS simulation.

Profiles of rotational and translational temperature along the same radial plane are shown in Fig. (8). In comparing the ES-BGK rotational temperature profile with that from the DSMC simulation performed here, we find an overestimate of about $13 \%$ in the ES-BGK values near the axis, and a slight underestimate (less than 1\%) at the nozzle wall. The same general pattern is also observed in a comparison of translational temperature profiles, although the translational temperature is underpredicted in the ES-BGK simulation by roughly $3 \%$ at the wall. While some of these discrepancies are significant, the general trends shown in the DSMC results are qualitatively reproduced very well by the ES-BGK method: The rotational temperature is slightly higher than the translational temperature for $y / R_{w}<0.7$, with a sign reversal in the temperature difference beyond this point. At the nozzle wall, the translational temperature is almost fully accommodated to the wall temperature, while the rotational temperature is considerably lower.

The difference between translational and rotational temperatures at the wall in both the ES-BGK and DSMC results may be attributed to the wall collision model. All particle-wall collisions are diffuse, so that the translational component of average thermal energy for reflected particles corresponds to either the wall temperature or the local stagnation temperature as measured by the velocity of incident particles. As the wall and stagnation temperatures are nearly equal, we expect a very small difference between the wall surface temperature and the gas translational temperature at the wall. In contrast, the average post-collision rotational energy of particles involved in adiabatic diffuse reflection corresponds to the rotational component of the gas static temperature, not the stagnation temperature. Rotational energy accommodation occurs for only a small fraction of wall collisions, so there should be relatively little difference in the average rotational energy between incident and reflected particles. In low density regions where the rotational relaxation time is large compared to other characteristic time scales for the flow, a significant lag may develop between rotational and translational temperatures. As the region under consideration has both a large rotational relaxation time and a boundary condition which tends to disproportionately increase the translational temperature, the trends observed at the wall in Fig. (8) seem physically justified.

Note that the NS simulation does not account for rotational nonequilibrium, so only a single temperature for this simulation is shown in the figure. The NS temperature profile shows relatively poor agreement with all of the other results, and gives a gas temperature at the wall between the translational and rotational temperatures found using the other methods. A no-slip adiabatic boundary condition is used for the wall in the NS calculation, so some temperature discontinuity at the wall is expected.

10

American Institute of Aeronautics and Astronautics Approved for public release; distribution unlimited. 
Profiles of bulk axial velocity are shown in Fig. (9), again at the radial plane near the nozzle exit defined by $x / R_{t}$ $=18.7$. Velocities here are normalized by the most probable thermal speed at the stagnation temperature $\mathrm{S}_{\mathrm{o}}=$ $\left(2 \mathrm{RT}_{\mathrm{o}}\right)^{1 / 2}=422 \mathrm{~m} / \mathrm{s}$. We find nearly identical profiles for the ES-BGK and the two DSMC simulations, with a deviation between the three results of less than $5 \%$ at all points on the plot. The NS velocity profile shows excellent agreement with the other results at the centerline, but diverges significantly within a short distance from the axis. While the ES-BGK and DSMC simulations give a wall slip velocity equal to about $28 \%$ of the centerline velocity, the NS no-slip boundary condition results in a velocity of zero at the wall. If a Maxwell-type slip model (based on the velocity gradient at the wall) had been employed in the NS simulation, we expect that better agreement would be found between the NS velocity profile and those for the other methods. A slip model would however affect density and temperature profiles as well, so it is not clear that its inclusion would lead to a net gain in overall accuracy for the NS simulation of this flow.

Figure (10) displays the variation along the centerline of continuum breakdown parameters, based on the DSMC simulation performed for this study. As defined by Boyd et al., ${ }^{19}$ the local degree of thermal nonequilibrium may be roughly characterized by the maximum value of a "gradient length local" Knudsen number $\mathrm{Kn}_{\mathrm{GLL}}(\mathrm{Q})$, where

$$
\mathrm{Kn}_{\mathrm{GLL}}(\mathrm{Q})=\frac{\lambda}{\mathrm{Q}}\left|\frac{\partial \mathrm{Q}}{\partial \mathrm{s}}\right|
$$

and Q is set to either the density, translational temperature or bulk speed of the gas. The symbol $\lambda$ here denotes the mean free path, and $\partial \mathrm{Q} / \partial \mathrm{s}$ is the partial derivative of $\mathrm{Q}$ in the direction of maximum gradient. As radial derivatives of macroscopic flow quantities are by definition zero along the centerline, $\partial \mathrm{s}$ here is in the axial direction. Significant scatter is observed among data points in the figure, as a result of both statistical noise and subtractive cancellation error in the evaluation of the derivatives. Tenth order polynomial trendlines, based on a least-squares fit, are therefore used to more clearly show the general trends among the three different parameters.

As is generally expected for a near-adiabatic expansion flow, the parameter based on density $\operatorname{Kn}_{\mathrm{GLL}}(\rho)$ gives the largest value through nearly the entire range considered. Based on this parameter, we find a local maximum in the degree of nonequilibrium a short distance downstream of the throat. This maximum can be attributed to the large curvature of streamlines around the throat, and the associated weak compression wave mentioned in the discussion of Figs. (3) and (4). The flow then equilibrates somewhat before rarefaction effects again become more prominent further downstream. A maximum $\operatorname{Kn}_{\mathrm{GLL}}(\rho)$ value of slightly less than 0.025 is ultimately reached in the nearfield plume region just upstream of the outflow boundary. Trends in this parameter are found to correlate well with the general level of agreement between ES-BGK and DSMC results as shown in Figs. (3) through (6). As the value of $\mathrm{Kn}_{\mathrm{GLL}}(\rho)$ increases with downstream distance along the centerline, translational and rotational temperatures are increasingly overpredicted by the ES-BGK method, while density and Mach number are underpredicted.

Note that the comparison of ES-BGK simulation results with DSMC, NS and experimental data is intended to assess the validity of the assumptions and approximations behind the method itself, and not to evaluate the tradeoff between efficiency and accuracy at larger time steps where advantages of the ES-BGK method over DSMC become apparent. Further study of time discretization errors on a simpler transition-regime flow is warranted to more clearly show the potential advantages of this method. However, if computational expense is measured as the mean calculation time per particle per time step at steady state, the ES-BGK simulation presented here is about $9 \%$ less expensive than the DSMC simulation. While this figure may seem discouraging, no optimization for efficiency is attempted here through either local or global variation in the time step size. Instead, a single time step size is used throughout the grid domain following the minimum mean collision time at the nozzle throat. As a result of the large ratio of mean collision time to time step size through much of the flowfield, a relatively small fraction of the total simulation time is taken up by collision operations. Given this fact, a $9 \%$ increase in overall efficiency does make the proposed method seem a promising alternative to DSMC for flows involving moderate rarefaction effects where collision operations account for a larger fraction of the total simulation time.

As the time step and cell size increase, some additional loss of accuracy is expected in the ES-BGK results, due to an error associated with the temporal and spatial decoupling of collision and advection processes in the gas, as well as a nonphysical equilibration of flow properties in each cell and an increase in numerical diffusion effects. ${ }^{20}$ At the same time, the computational expense of collision procedures will approach an asymptotic limit where all particle velocity and rotational energy values are reassigned during each time step. In contrast, DSMC collision procedures either give roughly linear scaling of computational expense with time step size, or permit the occurrence of nonphysical collisions for which the collision probability is greater than one. Alternate DSMC-based approaches

American Institute of Aeronautics and Astronautics Approved for public release; distribution unlimited. 
such as collision limiters ${ }^{21}$ typically require many times more operations under these conditions than procedures, such as those presented here, which involve direct sampling of particle properties from known distributions.

\section{Conclusions}

A method has been presented for the simulation of rarefied gas flows, based on a probabilistic solution to the ESBGK model of the Boltzmann equation. Methods described by Macrossan ${ }^{6}$ and Gallis and Torczynski ${ }^{8}$ were extended to allow for the consideration of a gas mixture involving diatomic species, and a procedure has been outlined to enforce exact momentum and energy conservation.

Results from an ES-BGK simulation were compared with DSMC, NS and experimental data for a nozzle flow of the type found in cold-gas spacecraft thrusters. Generally good quantitative agreement was observed between ESBGK, DSMC and experimental data for several flow properties, and the ES-BGK flowfield solution was shown to incorporate thermal nonequilibrium effects which were consistent with DSMC results. Based on this test case, we find the overall accuracy of the ES-BGK method to be superior to a traditional NS implementation but less than that of DSMC for the simulation of a rarefied expansion flow involving a simple diatomic gas. In general, the proposed method was shown to retain the accuracy of a numerical solution to the NS equations in the Knudsen number range where these equations may be assumed valid, and at higher Knudsen numbers accounts for effects of moderate thermal nonequilibrium that are neglected in the standard NS formulation.

While the ES-BGK method is built on a series of near-equilibrium approximations that may produce large errors in the simulation of highly rarefied flows, it avoids the calculation of individual molecular collisions which generally makes DSMC impractical for calculations involving very low Knudsen number flows. As particle movement, boundary conditions and sampling procedures are the same in the ES-BGK method as in DSMC, the method is ideally suited to implementation in a hybrid code for the simulation of flows involving a range of Knudsen number regimes. This avoids much of the complexity inherent in NS/DSMC hybrid codes, and reduces the sensitivity of computed flow characteristics to the interface location.

An optimization of simulation parameters to balance accuracy and efficiency is out of the scope of this study, but the lack of individual collision calculations in the ES-BGK method should allow for computational efficiency significantly greater than DSMC in near-equilibrium regions where a relatively large time step can be used. The overall efficiency will however be comparable to DSMC in regions with a higher degree of nonequilibrium, where assumptions underlying the method are likely valid only if the time step size is restricted to the local mean collision time. This method therefore holds promise as a compromise in both accuracy and efficiency between NS and DSMC, and may be a possible alternative to NS solvers in a hybrid code with DSMC, or to continuum methods based on higher-order moments of the Boltzmann equation for the simulation of transition regime flows where assumptions underlying the NS formulation may break down but DSMC is prohibitively expensive.

\section{Acknowledgments}

The authors gratefully acknowledge the Air Force Research Laboratory at Edwards Air Force Base for financial support of this work, with Tom Smith as technical monitor.

\section{References}

${ }^{1}$ Bird, G. A., Molecular Gas Dynamics and the Direct Simulation of Gas Flows, Clarendon Press, Oxford, 1994.

${ }^{2}$ Boyd, I. D., Penko, P. F., Meissner, D. L., and DeWitt, K. J., "Experimental and Numerical Investigations of Low-Density Nozzle and Plume Flows of Nitrogen," AIAA Journal, Vol. 30, No. 10, 1992, pp. 2453-2461.

${ }^{3}$ Montanero, J. M., Santos, A., Duffy, J. W., and Lutsko, J. F., "Stability of Uniform Shear Flow," Physical Review E, Vol. 57, No.1, 1998, pp. 546-556.

${ }^{4}$ Bruno, D., and Longo, S., "Monte Carlo Simulation of Nearly Kinematic Shock Fronts in Rarefied Gases," European Physical Journal, Applied Physics, Vol. 17, 2002, pp. 233-241.

${ }^{5}$ Bruno, D., Capitelli, M., and Longo, S., "A BGK/MC Method for the Simulation of Shock Waves in Binary Mixtures," AIAA Paper 2002-2895, 2002.

${ }^{6}$ Macrossan, M. N., "A Particle Simulation Method for the BGK Equation," Proceedings of the $22^{\text {nd }}$ International Symposium on Rarefied Gas Dynamics , American Institute of Physics, 2001, pp. 426-433.

${ }^{7}$ Macrossan, M. N., "v-DSMC: A Fast Simulation Method for Rarefied Flow," Journal of Computational Physics, Vol. 173, 2001, pp. 600-619.

${ }^{8}$ Gallis, M. A., and Torczynski, J. R., "The Application of the BGK Model in Particle Simulations," AIAA Paper 2000-2360, 2000.

12

American Institute of Aeronautics and Astronautics Approved for public release; distribution unlimited. 
${ }^{9}$ Andries, P., Bourgat, J., Le Tallec, P., and Perthame, B., "Numerical Comparison Between the Boltzmann and ES-BGK Models for Rarefied Gases," Institut National de Recherche en Informatique et en Automatique, Report No. 3872, 2000.

${ }^{10}$ Holway, L. H., "Kinetic Theory of Shock Structure Using an Ellipsoidal Distribution Function," Proceedings of the Fourth International Symposium on Rarefied Gas Dynamics, Academic Press, New York, 1966, pp. 193-215.

${ }^{11}$ Cercignani, C., The Boltzmann Equation and its Applications, Applied Mathematical Sciences, Springer Verlag, 1988.

${ }^{12}$ Andries, P., and Perthame, B., "The ES-BGK Model Equation with Correct Prandtl Number," Proceedings of the $22^{\text {nd }}$ International Symposium on Rarefied Gas Dynamics, American Institute of Physics, 2001, pp. 426-433.

${ }^{13}$ Sun, Q., and Boyd, I. D., "Evaluation of Macroscopic Properties in the Direct Simulation Monte Carlo Method," Journal of Thermophysics and Heat Transfer, Vol. 19, No. 3, 2005, pp. 329-335.

${ }^{14}$ Parker, J. G., "Rotational and Vibrational Relaxation in Diatomic Gases," Physics of Fluids, Vol. 2, 1959, pp. 449-462.

${ }^{15}$ Boyd, I. D., "Analysis of Rotational Noequilibrium in Standing Shock Waves of Nitrogen," AIAA Journal, Vol. 28, No. 11, 1990, pp. 1997-1999.

${ }^{16}$ Dietrich, S., and Boyd, I. D., "Scalar and Parallel Optimized Implementation of the Direct Simulation Monte Carlo Method," Journal of Computational Physics, Vol. 126, 1996, pp. 328-342.

${ }^{17}$ Rothe, D. E., "Electron-Beam Studies of Viscous Flow in Supersonic Nozzles," AIAA Journal, Vol. 9, No. 5, 1971, pp. 804-811.

${ }^{18}$ Chung, C., Kim, S. C., Stubbs, R. M., and De Witt, K. J., "Low Density Nozzle Flow by the Direct Simulation Monte Carlo and Continuum Methods," Journal of Propulsion and Power, Vol. 11, No. 1, 1995, pp. 64-70.

${ }^{19}$ Boyd, I. D., Chen, G., and Candler, G. V., "Predicting Failure of the Continuum Fluid Equations in Transitional Hypersonic Flows," Physics of Fluids, Vol. 7, No. 1, 1995, pp. 210-219.

${ }^{20}$ Breuer, K. S., Piekos, E. S., and Gonzales, D. A., "DSMC Simulations of Continuum Flows," AIAA Paper 952088, 1995.

${ }^{21}$ Bartel, T. J., Sterk, T. M., Payne, J. L., and Preppernau, P., "DSMC Simulation of Nozzle Expansion Flow Fields," AIAA Paper 94-2047, 1994.

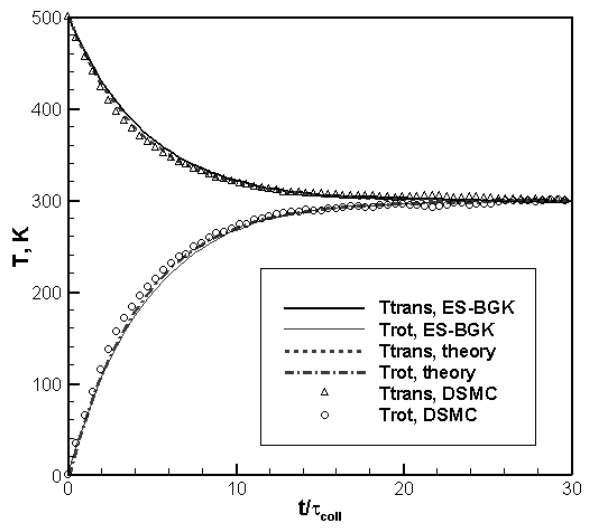

Fig. 1 Time variation in temperatures for rotational relaxation.

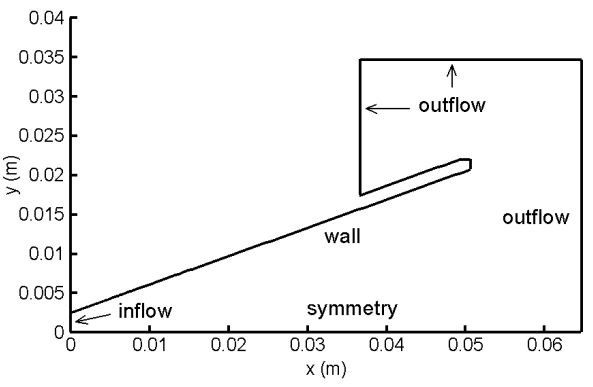

Fig. 2 Grid geometry for ES-BGK and DSMC simulations. 


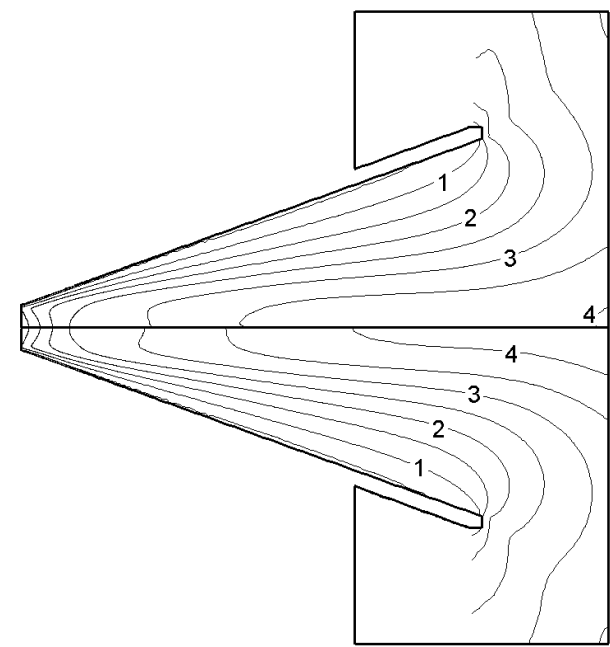

Fig. 3 Mach number contours for ES-BGK (top) and DSMC (bottom).

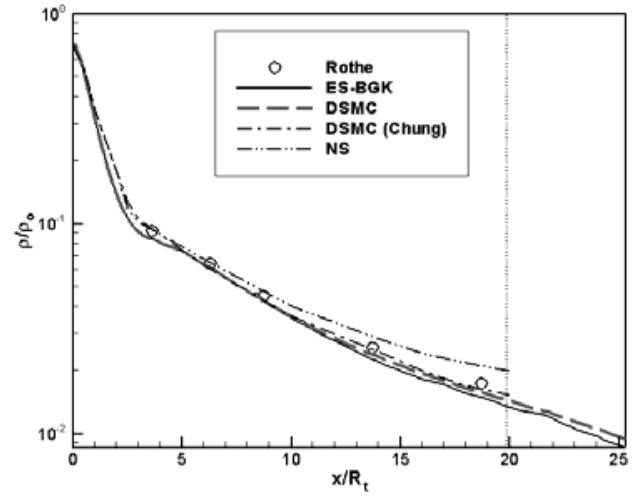

Fig. 5 Density variation along the nozzle centerline.

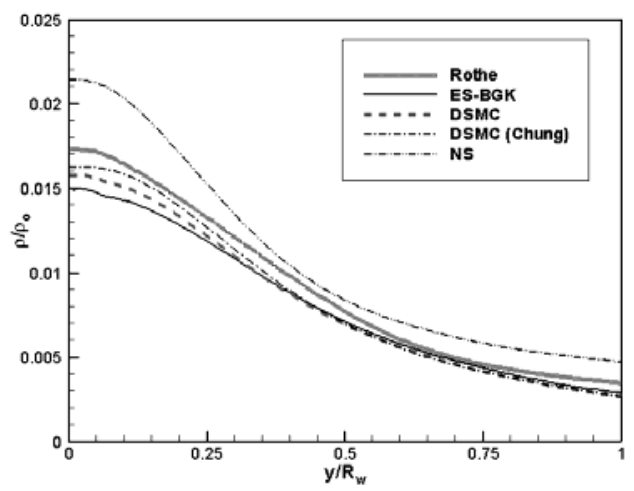

Fig. 7 Density variation along a radial plane at $\mathbf{x} / \mathbf{R}_{\mathbf{t}}=\mathbf{1 8 . 7}$.

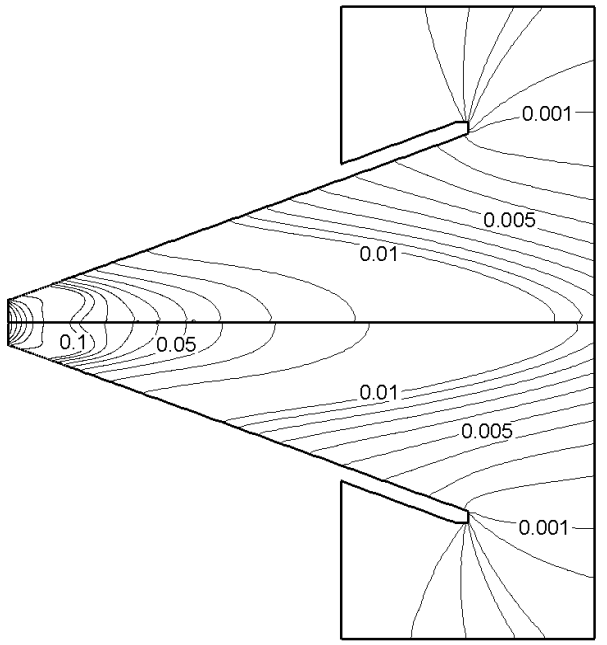

Fig. 4 Density contours for ES-BGK (top) and DSMC (bottom). Values are normalized by the stagnation density.

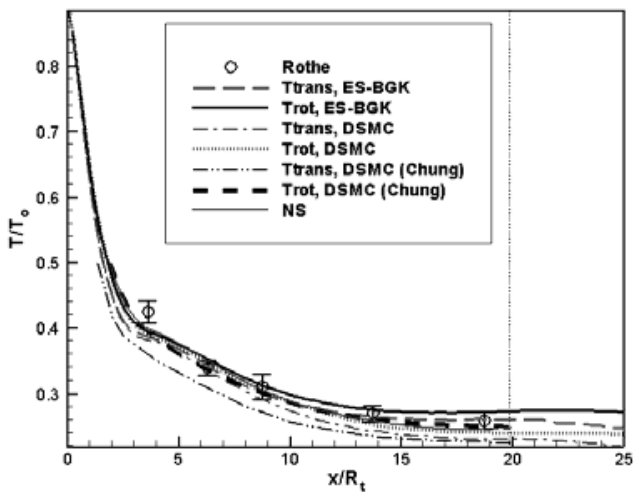

Fig. 6 Variation of rotational and translational temperature along the centerline.

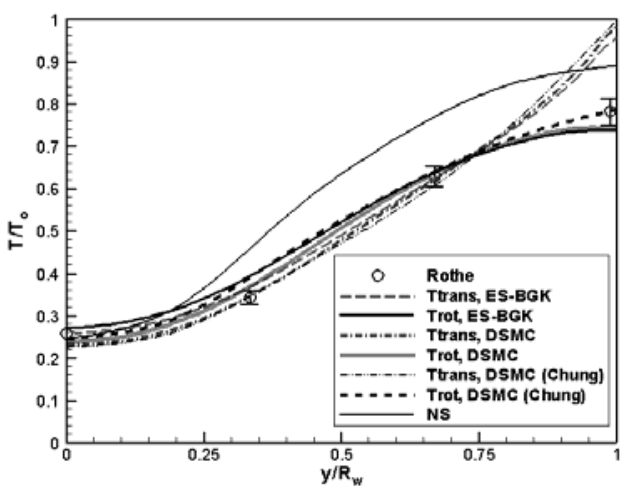

Fig. 8 Temperature variation along a radial plane at $\mathrm{x} / \mathbf{R}_{\mathrm{t}}=18.7$. 


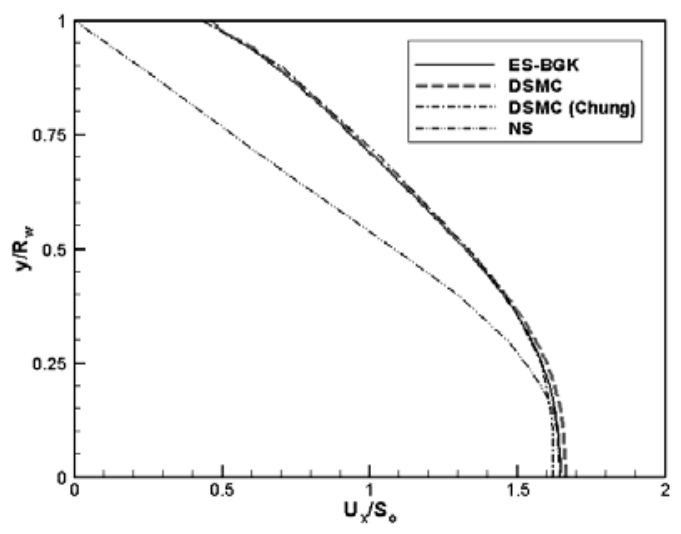

Fig. 9 Axial velocity profiles at $\mathbf{x} / \mathbf{R}_{\mathbf{t}}=\mathbf{1 8 . 7}$.

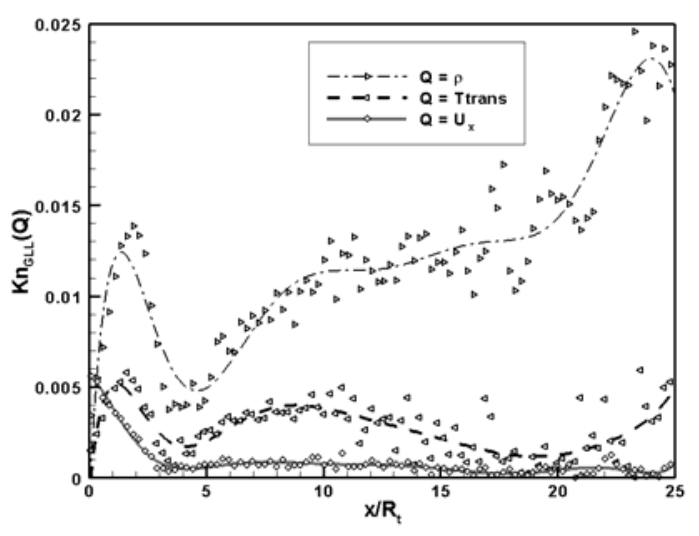

Fig. 10 Continuum breakdown parameters along the nozzle centerline. 